\title{
Association between Patient-Reported Outcomes and Survival in Patients with Advanced Urothelial Carcinoma Treated with Atezolizumab
}

\author{
Eugene Tan ${ }^{\mathrm{a}, 1}$, Ahmad Y. Abuhelwa ${ }^{\mathrm{a}, 1}$, Sarah Badaoui ${ }^{\mathrm{a}}$, Natansh D. Modi ${ }^{\mathrm{a}}$, Michael D. Wiese ${ }^{\mathrm{b}}$, \\ Ross A. McKinnon ${ }^{\mathrm{a}}$, Michael J. Sorich ${ }^{\mathrm{a}}$ and Ashley M. Hopkins ${ }^{\mathrm{a}, *}$

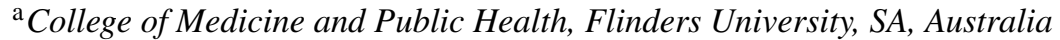 \\ ${ }^{\mathrm{b}}$ Health and Biomedical Innovation, UniSA: Clinical and Health Sciences, University of South Australia, \\ Adelaide, SA, Australia
}

Received 23 September 2021

Accepted 13 December 2021

Pre-press 27 December 2021

Published 11 March 2022

\begin{abstract}
.
BACKGROUND: Atezolizumab is an immune checkpoint inhibitor (ICI) and a frontline treatment of patients with cisplatinineligible advanced urothelial carcinoma (UC). There is limited evidence on the prognostic value of patient reported outcomes (PROs) in advanced UC treatment, particularly in the context of ICI therapy.

OBJECTIVE: To investigate the prognostic association of PROs with survival in patients with advanced UC treated with atezolizumab.

METHODS: This study used data from 467 patients with advanced UC initiating atezolizumab in the IMvigor211 trial. Pre-treatment PROs association with overall survival (OS) and progression free survival (PFS) was assessed using Cox proportional hazard analysis. PROs were recorded via the European Organisation for Research and Treatment of Cancer QLQ-C30. Discrimination performance was assessed via the C-statistic $(c)$.

RESULTS: Patient reported physical function, pain, appetite loss, global health, fatigue, role function, constipation, nausea and vomiting, dyspnoea, and insomnia were significantly associated with OS and PFS on univariable and adjusted analysis $(P<0.05)$. Physical function $(c=0.63)$, pain $(c=0.63)$, appetite loss $(c=0.62)$, global health status $(c=0.62)$, and fatigue $(c=0.62)$, were the most prognostic factors of OS. The OS discrimination performance of physical function $(c=0.61)$ was superior to ECOG PS $(c=0.58)$. Of patients assessed by investigators as having no performance restrictions (ECOG PS of $0), 38(18 \%)$ and $91(42 \%)$ self-reported low and intermediate physical function scores, respectively.

CONCLUSION: Pre-treatment PROs were identified as independent prognostic factors of OS and PFS. Patient-reported physical function was more prognostic of OS than ECOG PS. This highlights a potential for PROs to enable improved patient stratification in ICI trials.
\end{abstract}

Keywords: Patient-reported outcome, overall survival, urothelial cancer, atezolizumab

\footnotetext{
${ }^{1}$ Equal first-author.

${ }^{*}$ Correspondence to: Dr. Ashley Hopkins, Room 5E315, Flinders Medical Centre, Bedford Park, Adelaide, SA, 5042, Australia. Tel.: +61 88201 5647; E-mail: ashley.hopkins@ flinders.edu.au.
} 


\section{INTRODUCTION}

Atezolizumab is an immune checkpoint inhibitor (ICI) and an important emerging treatment option for advanced urothelial carcinoma (UC), particularly in patients who are ineligible for or resistant to platinum-containing therapies [1,2]. To date, there is no reliable marker to accurately predict patients who will benefit most from ICIs in advanced UC, and there is significant variability in response between patients [3, 4]. This knowledge gap impedes trial design, shared decision-making, and precision medicine.

Patient-reported outcomes (PROs) are measures of quality of life, emotional, financial, and functional status that are assessed by self-reported tools from a patient's perspective [5]. With major international oncology societies highlighting the potential benefits of PROs in facilitating shared decision-making and patient empowerment [6-8], much research is aimed towards unlocking their full potential. Furthermore, recent studies have demonstrated the prognostic value of PROs in patients with a variety of advanced cancers [9-12]. Eastern Cooperative Oncology Group-performance status (ECOG-PS) is a clinician interpreted measure of physical function that is routinely used in oncology trial design, and several studies have demonstrated that patientreported physical function potentially has greater prognostic value [12-17]. However, there is limited evidence on the value of PROs in advanced UC treatment, particularly in the context of ICI therapy. We hypothesize that PROs are prognostic of survival outcomes in patients with advanced UC initiating immunotherapy. This study aims to investigate the association of PROs with survival outcomes in patients with advanced UC treated with atezolizumab. Exploratory analysis will also be conducted to establish if pre-treatment physical function is more prognostic of survival than ECOG-PS.

\section{MATERIALS AND METHODS}

\section{Patient population}

This study was a secondary analysis of individual participant data (IPD) from phase 3 randomized controlled trial (RCT) IMvigor211 (NCT02302807) [1]. Briefly, IMvigor211 randomized participants $1: 1$ to 3 weekly doses of either atezolizumab $(1,200 \mathrm{mg})$ or chemotherapy (investigator's choice of vinflunine $\left(320 \mathrm{mg} / \mathrm{m}^{2}\right)$, paclitaxel $\left(175 \mathrm{mg} / \mathrm{m}^{2}\right)$ or docetaxel $\left.\left(75 \mathrm{mg} / \mathrm{m}^{2}\right)\right)$ [1, 3]. Key inclusion criteria for IMvigor 211 included age $\geq 18$, evaluable UC disease, ECOG-PS $\leq 1$, availability of a sample for PD-L1 evaluation, have received $\leq 2$ lines of prior therapy, experienced disease progression with platinum-containing therapy, and no prior ICI therapy [1].

Data was accessed according to Hoffmann-La Roche policy and has been made available through Vivli, Inc (www.vivli.org). Secondary analysis of de-identified IPD was classified as minimal risk research and was confirmed exempt from review by the Southern Adelaide Local Health Network, Office for Research and Ethics.

\section{Predictors and outcomes}

The primary evaluated outcome was overall survival (OS). Progression-free survival (PFS), objective response and treatment-related any adverse events (AEs) of grade $\geq 3$ were assessed as exploratory outcomes. OS was defined as the time from randomization to death due to any cause $[1,18]$. PFS was defined as the time from randomization to either the first event of disease progression or death from any cause. Progression and objective response were determined by the investigator per RECIST v1.1 $[1,18]$. AEs were graded per the National Cancer Institute's Common Terminology Criteria for Adverse Events, version 4.0 (NCI CTCAE v4.0).

PROs were recorded at baseline, on day one of each treatment cycle, and at 30 days after the last treatment dose (up to approximately 25 months) via the European Organisation and Treatment of Cancer Quality of Life Questionnaire Core 30 (EORTC QLQ-C30) version 3.0. [19]. The questionnaire enables the calculation of functional scale (cognitive, role, physical, emotional, and social), symptom-related scale (fatigue, pain, nausea, and vomiting), and global health status scores [19]. For functional scales, a higher score indicates the patient's perception of "better" functioning. Whilst for symptom-related scales and measures, a higher score indicates the patient's perception of "worse" symptoms [20]. The primary evaluated predictors in this study were pretreatment PRO measures of function, symptoms, and global health status.

\section{Statistical analysis}

Cox proportional hazard analysis was used to assess the association between each pre-treatment PRO with OS, PFS, and AEs. Results are reported as hazard ratios (HR) with $95 \%$ confidence intervals 
Table 1

Univariable and adjusted association between PROs and overall survival for patients treated with atezolizumab

\begin{tabular}{|c|c|c|c|c|c|c|c|c|c|}
\hline & \multicolumn{5}{|c|}{ Univariable } & \multicolumn{4}{|c|}{ Adjusted** } \\
\hline & $\mathrm{n}$ & $\mathrm{HR}^{*}$ & $95 \% \mathrm{CI}$ & $P$-value & $c$ & $\mathrm{n}$ & $\mathrm{HR}^{*}$ & $95 \% \mathrm{CI}$ & $P$-value \\
\hline Physical function & 441 & 0.84 & 0.80 to 0.88 & $<0.001$ & 0.63 & 345 & 0.87 & 0.81 to 0.93 & $<0.001$ \\
\hline Pain & 441 & 1.15 & 1.11 to 1.19 & $<0.001$ & 0.63 & 345 & 1.09 & 1.04 to 1.15 & $<0.001$ \\
\hline Appetite loss & 440 & 1.15 & 1.11 to 1.19 & $<0.001$ & 0.62 & 345 & 1.13 & 1.08 to 1.19 & $<0.001$ \\
\hline Global health & 439 & 0.84 & 0.80 to 0.88 & $<0.001$ & 0.62 & 344 & 0.87 & 0.81 to 0.93 & $<0.001$ \\
\hline Fatigue & 441 & 1.15 & 1.10 to 1.20 & $<0.001$ & 0.61 & 345 & 1.11 & 1.05 to 1.18 & $<0.001$ \\
\hline Role function & 441 & 0.90 & 0.87 to 0.94 & $<0.001$ & 0.6 & 345 & 0.95 & 0.90 to 1.00 & 0.040 \\
\hline Social function & 441 & 0.90 & 0.86 to 0.94 & $<0.001$ & 0.58 & 345 & 0.96 & 0.91 to 1.02 & 0.172 \\
\hline Emotional function & 441 & 0.90 & 0.85 to 0.95 & $<0.001$ & 0.58 & 345 & 0.95 & 0.89 to 1.02 & 0.130 \\
\hline Constipation & 439 & 1.08 & 1.04 to 1.11 & $<0.001$ & 0.58 & 343 & 1.05 & 1.00 to 1.09 & 0.029 \\
\hline Nausea and vomiting & 441 & 1.23 & 1.15 to 1.31 & $<0.001$ & 0.57 & 345 & 1.23 & 1.14 to 1.33 & $<0.001$ \\
\hline Dyspnoea & 440 & 1.08 & 1.04 to 1.13 & $<0.001$ & 0.56 & 344 & 1.10 & 1.04 to 1.16 & $<0.001$ \\
\hline Cognitive function & 441 & 0.90 & 0.85 to 0.95 & $<0.001$ & 0.56 & 345 & 0.94 & 0.88 to 1.01 & 0.084 \\
\hline Insomnia & 440 & 1.08 & 1.04 to 1.12 & $<0.001$ & 0.56 & 344 & 1.08 & 1.03 to 1.14 & $<0.001$ \\
\hline Financial difficulties & 440 & 1.04 & 1.00 to 1.08 & 0.066 & 0.53 & 344 & 1.00 & 0.95 to 1.05 & 0.859 \\
\hline Diarrhoea & 440 & 1.06 & 1.00 to 1.12 & 0.041 & 0.52 & 345 & 0.98 & 0.91 to 1.06 & 0.660 \\
\hline
\end{tabular}

$\mathrm{CI}=$ confidence interval, $\mathrm{HR}=$ hazard ratio, $n=$ number of patients. ${ }^{*} \mathrm{HR}$ based on 10 -unit increase. ${ }^{* *}$ Adjusted for haemoglobin, sex, age, race, smoking history, ECOG performance status, disease status, PD-L1 tumor-infiltrating immune cell expression level, time since diagnosis, number of prior systemic therapies in advanced setting, liver metastasis, bone metastasis, lung metastasis, lactate dehydrogenase and neutrophil to lymphocyte ratio. A total of 120 patients had missing data in the adjustment variables.

(95\% CI). A threshold of $p<0.05$ was used to determine statistical significance. Complete case analyses were conducted. Discrimination performance was assessed using the concordance statistic (C-statistic). Continuous variables were explored for potential non-linear associations. The best model fit was determined using the Akaike information criterion (AIC) and visual checks. Analyses were adjusted for race, sex, age, smoking status, ECOG PS, stage, presence of liver metastasis, haemoglobin concentration, PD-L1 expression, time since diagnosis, number of prior treatments, neutrophil to lymphocyte ratio and lactate dehydrogenase concentration. The discrimination performance of PROs with ECOG PS were evaluated. Exploratory analyses of the association of PROs with survival within the chemotherapy cohorts were conducted. Associations between pre-treatment PROs and objective response were assessed via logistic regression.

Kaplan-Meier analysis was used to visually represent the associations between significant PROs with OS and PFS. For these plots, PROs were classified as "low", "intermediate" and "high" based on the interquartile range (IQR) presented in the EORTC QLQ-C30 reference value manual for the "All cancer patients: recurrent/metastatic" population. Low, intermediate, and high groups were respectively defined by the lower 25 th, the 25 th to 75 th, and the upper 25th percentiles according to the EORTC QLQ-C30 reference IQR [19]. All analyses were performed using $\mathrm{R}$ version 3.6.2.

\section{RESULTS}

\section{Patient population}

Data were available from 931 participants within the IMvigor211 of which 467 patients were randomized to atezolizumab treatment. Of the 467 patients in the randomised atezolizumab arm, 459 received actual atezolizumab treatment (Supplementary Table 1 and Supplementary Table 2). Depending on the evaluated PRO outcome, 18 to 20 of the 459 patients had missing PRO data (Supplementary Table 2). Median follow-up was 17 [17-19] months in the atezolizumab cohort. There were 318 and 401 OS and PFS events in the atezolizumab cohort, respectively.

\section{Prognostic associations of PROs with overall survival}

Based upon the AIC and visual inspections, linear transformations best described the associations between pre-treatment PROs and OS. Univariable and adjusted analyses identified significant associations for physical function, pain, appetite loss, global health, fatigue, role function, constipation, nausea and vomiting, dyspnoea, and insomnia with OS outcomes $(P<0.05$, Table 1). Of the assessed PROs, physical function $(c=0.63)$, pain $(c=0.63)$, appetite loss $(c=0.62)$, global-health $(c=0.62)$, and fatigue $(c=0.62)$, were the most prognostic for OS (Table 1). Figure 1 presents Kaplan-Meier estimates of OS 

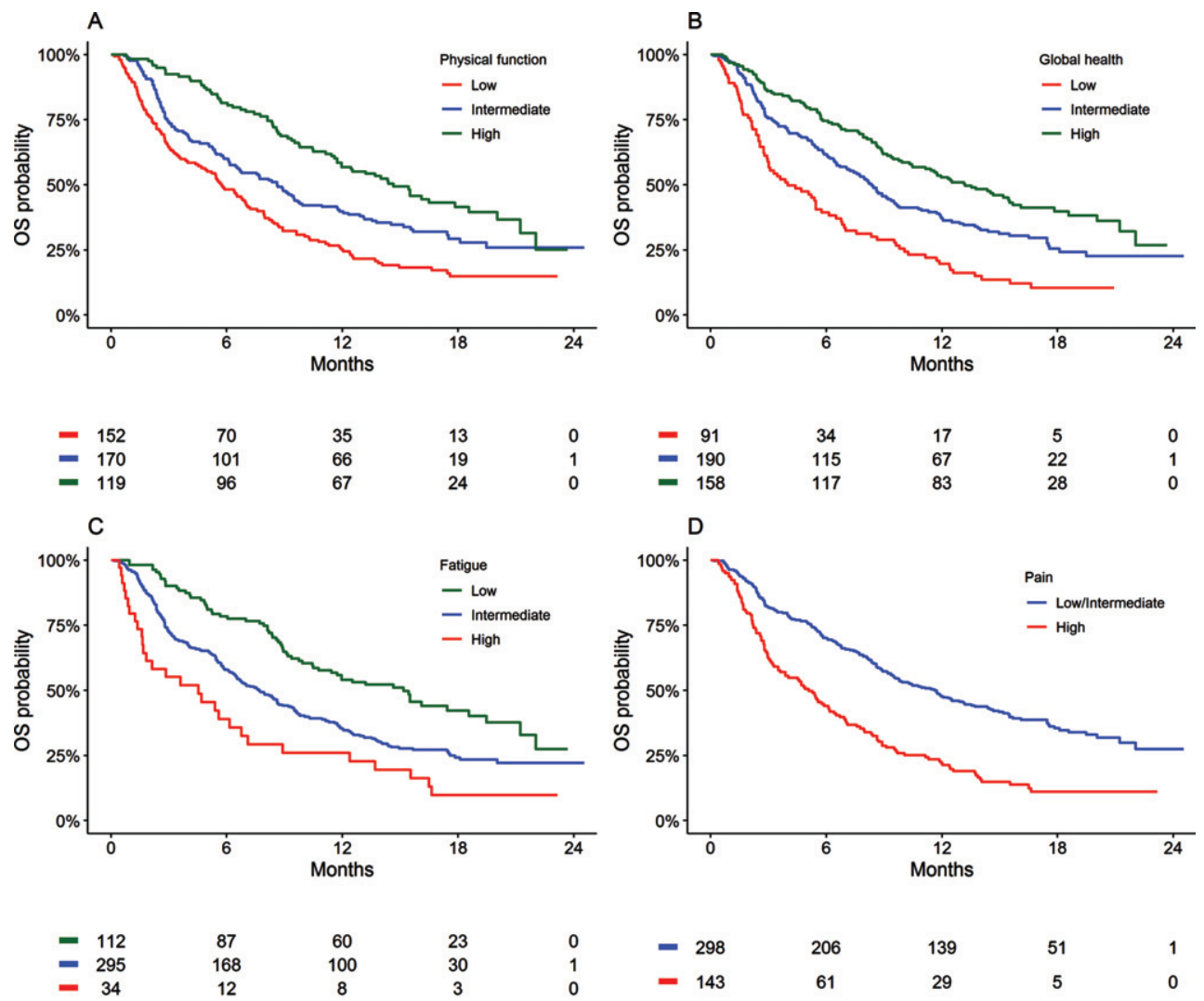

$\begin{array}{cccc}206 & 139 & 51 & 1 \\ 61 & 29 & 5 & 0\end{array}$

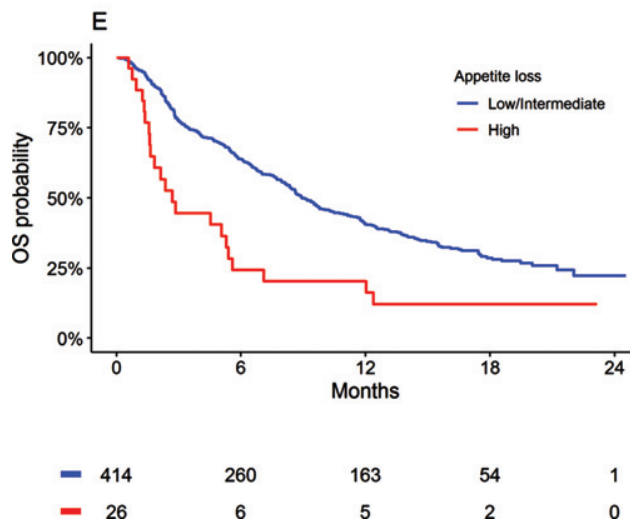

Fig. 1. Kaplan-Meier estimates of overall survival (OS) by patient-reported physical function, fatigue, appetite, global health, and pain symptoms for patients treated with atezolizumab.

according to pre-treatment physical function, pain, appetite loss, global-health status, and fatigue within the atezolizumab treated cohort.

Physical function, pain, appetite loss, global health, and fatigue were significantly associated with PFS on univariable and adjusted analysis $(P<0.05$, Supplementary Table 3 and Supplementary Figure 1). Similar associations were identified between objective response and PROs on univariable analysis
(Supplementary Table 4). Physical function, pain, appetite loss, and fatigue were also significantly associated with grade $\geq 3$ adverse effects on univariable analysis (Supplementary Table 5)

Supplementary Table 6 demonstrates that physical function, pain, appetite loss, global health, and fatigue were significantly associated with OS on univariable and adjusted analysis within the control arm (i.e., these PROs were also prognostic in the patient 
Table 2

Univariable and multivariable association between patient-reported physical function and ECOG-PS with overall survival for atezolizumab patient cohort within IMvigor211

\begin{tabular}{|c|c|c|c|c|c|c|c|c|c|}
\hline & \multicolumn{5}{|c|}{ Univariable } & \multicolumn{4}{|c|}{ Multivariable model ${ }^{* *}$} \\
\hline & $\mathrm{n}$ & $\mathrm{HR}^{*}$ & $95 \% \mathrm{CI}$ & $P$-value & $c$ & $\mathrm{n}$ & $\mathrm{HR}^{*}$ & $95 \% \mathrm{CI}$ & $P$-value \\
\hline Physical function status & & & & $<0.001$ & 0.61 & & & & $<0.001$ \\
\hline High & 119 & 1.00 & & & & 119 & 1.00 & & \\
\hline Intermediate & 170 & 1.56 & 1.16 to 2.10 & & & 170 & 1.50 & 1.11 to 2.02 & \\
\hline Low & 152 & 2.40 & 1.79 to 3.23 & & & 152 & 2.12 & 1.56 to 2.89 & \\
\hline ECOG PS Score & & & & $<0.001$ & 0.58 & & & & 0.003 \\
\hline 0 & 216 & 1.00 & & & & 207 & 1.00 & & \\
\hline 1 & 243 & 1.65 & 1.32 to 2.07 & & & 234 & 1.43 & 1.13 to 1.81 & \\
\hline
\end{tabular}

$\mathrm{CI}=$ confidence interval, $\mathrm{HR}=$ hazard ratio. ${ }^{*} \mathrm{HR}$ based on 10 -unit increase. ${ }^{* *}$ Multivariable model testing if there is independent information from both patient reported physical function and ECOG PS.

cohort treated with chemotherapy). Similar associations were identified for PFS, other than for physical function where the association did not reach statistical significance on adjusted analysis (Supplementary Table 7)

\section{Comparison of patient-reported physical function with ECOG PS}

As patient-reported physical function was the best performing PRO for predicting OS prognosis, and ECOG PS is a commonly used physician-assessment of physical function, this study compared the discriminative performance of patient-reported physical function and ECOG PS (analysis are based upon prior studies $[12,13])$.

In the cohort of 441 participants treated with atezolizumab, 152 (34\%), 170 (39\%), 119 (27\%) respectively reported physical function in the low, intermediate, and high EORTC QLQ-C30 reference quartiles (Supplementary Table 8). Clinicians defined 216 participants as having an ECOG PS of 0 , and 243 as an ECOG PS of 1. Notably, of the 216 participants assessed by the investigator have an ECOG PS of 0 (fully active; no performance restrictions), $60 \%$ of participants self-reported physical function limitations - $38(18 \%)$ reported low physical function and 91 (42\%) reported intermediate physical function. Univariable analysis identified a significant relationship between patient-reported physical function groups and OS $(P<0.001)$, with a prognostic performance $(c)$ of 0.61 (Supplementary Table 7). Univariable analysis also identified a significant relationship between ECOG PS and OS $(P<0.001)$, with a prognostic performance $(c)$ of 0.58 - slightly less prognostic than patient-reported physical function (Table 2). Upon multivariable analysis, patient-reported physical function $(P<0.001)$ and ECOG PS $(P<0.003)$ were significantly asso- ciated with OS indicating that each is providing an independent prognostic information (Table 2). Similar findings were observed in the chemotherapy cohort (Supplementary Table 9).

\section{DISCUSSION}

This is the first study to demonstrate that pretreatment PROs are significantly associated with OS and PFS in patients with platinum-refractory advanced UC who initiate treatment with the ICI atezolizumab. Exploratory analysis suggests that patient-reported physical function may be more prognostic than ECOG-PS. This was driven by $60 \%$ of the cohort self-reporting low to intermediate physical function, despite clinicians categorising their condition as an ECOG PS of 0.

Recent research highlights the prognostic potential of PROs in several advanced cancer types [9-12], although studies have been limited by small sample sizes and few have investigated the potential significance in patients with advanced cancer who are initiating ICI therapies. This study demonstrated that patient-reported physical function, pain, appetite loss, global health, and fatigue were the most prognostic PROs for OS and PFS within patients with metastatic UC initiating atezolizumab. Confirming whether these results are generalizable to other ICIs, first-line ICI use, or ICI combination therapy is an important future direction of research.

ECOG PS is a well-established clinician-assessed measure of patient function and is widely used to stratify clinical trial cohorts and aid treatment-related decisions in the clinic [21]. However, there are other patient-related factors such as socioeconomic and psychological status may not be accounted for by the ECOG PS [22]. Previous studies have shown discordances between patient and clinician reported 
adverse effects in cancer clinical trials [23-25]. Interestingly, in the present analysis, patient-reported physical function was identified to be more prognostic of OS than ECOG PS, and 18 and $42 \%$ of patients classified as ECOG PS 0 reported low and intermediate physical function, respectively. This indicates that $60 \%$ of the patients who were clinician defined as 'fully active, and able to carry on all pre-disease performance without restrictions' had self-reported limitations in physical functioning. This finding suggests that use of structured questions/questionnaires may improve clinician assessment of a patients' performance status. This study goes on to demonstrate that patient-reported physical function and ECOG PS were independent prognostic factors for OS. This finding supports prior studies that demonstrated significant and independent prognostic value of patient-reported physician function for patients with lung [13] and breast cancers [12]. This highlights a potential for patient-reported physician function to be used as a stratification factor in ICI clinical trials. Minimally, reporting of baseline patient-reported physician function scores would provide important patient context to clinical trial reports and publications.

Limitations of this study include the post-hoc, exploratory nature which introduces implications on statistical powers and type I errors; while the retrospective design introduces an inability to adjust for unmeasured confounders or to evaluate alternate prognostic parameters (e.g. data from wearable devices or alternate PRO tools). Another potential limitation of this study is that the identified associations were restricted to patients treated with atezolizumab monotherapy within a clinical trial. Stringent inclusion criteria of clinical trials can limit findings generalizability to real-world populations. For example, none of the patients included in the trial had an ECOG PS of greater than 1. Future research should therefore be directed towards evaluating PROs in real-world populations with a broader ECOG PS distribution. Future research should also consider investigating the associations between comorbidity measures (e.g., Charlson, Exhauster), wearable devices data, and PROs and evaluate their potential to be combined to predict likely treatment outcomes. Further, as both patient-reported physical function and ECOG PS contain independent prognostic information, building multivariable prognostic tools with the range of PRO domains in conjunction with ECOG PS is an interesting future direction of research.
In conclusion, pre-treatment PROs were identified as independent prognostic factors of OS and PFS in patients with advanced UC initiated on atezolizumab. Patient-reported physical function, pain, appetite loss, global health, and fatigue were most prognostic for OS and PFS. Patient-reported physical function was more prognostic for OS than ECOG PS. This study highlights the potential for PROs to be used as a stratification factor for ICI clinical trials and to inform disease prognosis.

\section{ACKNOWLEDGMENTS}

This publication is based on research using de-identified individual participant data from data contributor Hoffmann-La Roche that has been made available through Vivli, Inc. Vivli has not contributed to or approved, and is not in any way responsible for, the contents of this publication.

\section{FUNDING}

R.A.M, and M.J.S are supported by Beat Cancer Research Fellowships from Cancer Council South Australia. A.M.H is supported by a Postdoctoral Fellowship from the National Breast Cancer Foundation, Australia (PF-17-007). Data access and salary of A.Y.A is supported by funding from an Australian, Tour de Cure Early Career Research Grant (RSP-15518/19).

\section{AUTHORS CONTRIBUTIONS}

Conception

A.Y. Abuhelwa, Eugene Tan, S. Badaoui, A.M. Hopkins, M.J. Sorich

\section{Performance of work}

A.Y. Abuhelwa, Eugene Tan, S. Badaoui, A.M. Hopkins, M.J. Sorich

\section{Interpretation of data}

A.Y. Abuhelwa, E. Tan, S. Badaoui, A.M. Hopkins, M.J. Sorich, R.A. McKinnon, M. Wiese, N.D. Modi 


\section{Writing the article}

A.Y. Abuhelwa, Eugene Tan, Sarah Badaoui, A.M. Hopkins, M.J. Sorich, R.A. McKinnon, M. Wiese, N.D. Modi

\section{ETHICAL CONSIDERATIONS}

Secondary analysis of anonymized clinical trial data was confirmed negligible risk research by the Southern Adelaide Local Health Network, Office for Research and Ethics and was exempt from review.

\section{CONFLICTS OF INTEREST}

RAM and MJS report investigator-initiated project grants from Pfizer, outside the submitted work. AYA, ET, SB, NDM, MDW and AMH have no conflicts of interest to disclose.

\section{SUPPLEMENTARY MATERIAL}

The supplementary material is available in the electronic version of this article: https://dx.doi.org/ 10.3233/BLC-211613.

\section{REFERENCES}

[1] Powles T, Durán I, van der Heijden MS, Loriot Y, Vogelzang NJ, De Giorgi U, et al. Atezolizumab versus chemotherapy in patients with platinum-treated locally advanced or metastatic urothelial carcinoma (IMvigor211): a multicentre, open-label, phase 3 randomised controlled trial. Lancet. 2018;391(10122):748-57.

[2] Galsky MD, Arija JÁA, Bamias A, Davis ID, De Santis M, Kikuchi E, et al. Atezolizumab with or without chemotherapy in metastatic urothelial cancer (IMvigor 130): a multicentre, randomised, placebo-controlled phase 3 trial. The Lancet. 2020;395(10236):1547-57.

[3] Abuhelwa AY, Kichenadasse G, McKinnon RA, Rowland A, Hopkins AM, Sorich MJ. Machine Learning for Prediction of Survival Outcomes with Immune-Checkpoint Inhibitors in Urothelial Cancer. Cancers. 2021;13(9).

[4] Hopkins AM, Rowland A, Kichenadasse G, Wiese MD, Gurney H, McKinnon RA, et al. Predicting response and toxicity to immune checkpoint inhibitors using routinely available blood and clinical markers. British Journal of Cancer. 2017;117(7):913-20.

[5] Weldring T, Smith SMS. Patient-Reported Outcomes (PROs) and Patient-Reported Outcome Measures (PROMs). Health Serv Insights. 2013;6:61-8.

[6] Strong LE. The Past, Present, and Future of PatientReported Outcomes in Oncology. American Society of Clinical Oncology Educational Book. 2015(35):e616-e20.

[7] Kotronoulas G, Nora Kearney, Roma Maguire, Alison Harrow, David Di Domenico, Suzanne Croy, et al. What Is the Value of the Routine Use of Patient-reported Outcome Measures toward Improvement of Patient Outcomes, Processes of Care, and Health Service Outcomes in Cancer Care? A Systematic Review of Controlled Trials. Journal of Clinical Oncology. 2014;32(14):1480-501.

[8] Basch E, Barbera L, Kerrigan CL, Velikova G. Implementation of Patient-Reported Outcomes in Routine Medical Care. American Society of Clinical Oncology Educational Book. 2018(38):122-34.

[9] Mierzynska J, Piccinin C, Pe M, Martinelli F, Gotay C, Coens C, et al. Prognostic value of patient-reported outcomes from international randomised clinical trials on cancer: a systematic review. The Lancet Oncology. 2019; 20(12):e685-e98.

[10] Phippen NT, Secord AA, Wolf S, Samsa G, Davidson B, Abernethy AP, et al. Quality of life is significantly associated with survival in women with advanced epithelial ovarian cancer: An ancillary data analysis of the NRG Oncology/Gynecologic Oncology Group (GOG-0218) study. Gynecologic Oncology. 2017;147(1):98-103.

[11] Ediebah DE, Coens C, Zikos E, Quinten C, Ringash J, King MT, et al. Does change in health-related quality of life score predict survival? Analysis of EORTC 08975 lung cancer trial. British Journal of Cancer. 2014;110(10):2427-33.

[12] Badaoui S, Kichenadasse G, Rowland A, Sorich MJ, Hopkins AM. Patient-Reported Outcomes Predict ProgressionFree Survival of Patients with Advanced Breast Cancer Treated with Abemaciclib. The Oncologist.n/a(n/a).

[13] Hopkins AM, Wagner J, Kichenadasse G, Modi N, Rowland A, Sorich MJ. Patient-reported outcomes as a prognostic marker of survival in patients with advanced nonsmall cell lung cancer treated with immunotherapy. International Journal of Cancer. 2020;147(11):3085-9.

[14] Kerrigan K, Patel SB, Haaland B, Ose D, Weinberg Chalmers A, Haydell T, et al. Prognostic Significance of PatientReported Outcomes in Cancer. JCO Oncol Pract. 2020; 16(4):e313-e23.

[15] Quinten C, Coens C, Mauer M, Comte S, Sprangers MAG, Cleeland $\mathrm{C}$, et al. Baseline quality of life as a prognostic indicator of survival: a meta-analysis of individual patient data from EORTC clinical trials. The Lancet Oncology. 2009;10(9):865-71.

[16] Cella D, Traina S, Li T, Johnson K, Ho KF, Molina A, et al. Relationship between patient-reported outcomes and clinical outcomes in metastatic castration-resistant prostate cancer: post hoc analysis of COU-AA-301 and COU-AA302. Annals of Oncology. 2018;29(2):392-7.

[17] Husson O, de Rooij BH, Kieffer J, Oerlemans S, Mols F, Aaronson NK, et al. The EORTC QLQ-C30 Summary Score as Prognostic Factor for Survival of Patients with Cancer in the "Real-World": Results from the Population-Based PROFILES Registry. The oncologist. 2020;25(4):e722-e32.

[18] Piper M, Grant MD, Basch E, Oliansky D, N. A. Progression-Free Survival: What Does It Mean for Psychological Well-Being or Quality of Life? Rockville, MD 20850 Agency for Healthcare Research and Quality; 2013 April 2013. pp. 34.

[19] Aaronson NK, Ahmedzai S, Bergman B, Bullinger M, Cull A, Duez NJ, et al. The European Organization for Research and Treatment of Cancer QLQ-C30: A Qualityof-Life Instrument for Use in International Clinical Trials in Oncology. JNCI: Journal of the National Cancer Institute. 1993;85(5):365-76.

[20] Fayers PM, Aaronson NK, Bjordal K, Groenvold M, Curran D, Bottomley A. The EORTC QLQ-C30 Scoring Manual (3rdEdition). Brussels: European Organisation for Research and Treatment of Cancer; 2001. 
[21] Azam F, Latif MF, Farooq A, Tirmazy SH, AlShahrani S, Bashir S, et al. Performance Status Assessment by Using ECOG (Eastern Cooperative Oncology Group) Score for Cancer Patients by Oncology Healthcare Professionals. Case Rep Oncol. 2019;12(3):728-36.

[22] Datta SS, Ghosal N, Daruvala R, Chakraborty S, Shrimali RK, van Zanten C, et al. How do clinicians rate patient's performance status using the ECOG performance scale? A mixed-methods exploration of variability in decisionmaking in oncology. Ecancermedicalscience. 2019;13:913.

[23] Basch E, Iasonos A, McDonough T, Barz A, Culkin A, Kris $\mathrm{MG}$, et al. Patient versus clinician symptom reporting using the National Cancer Institute Common Terminology Criteria for Adverse Events: results of a questionnaire-based study. The Lancet Oncology. 2006;7(11):903-9.
[24] Fromme EK, Eilers KM, Mori M, Hsieh YC, Beer TM. How accurate is clinician reporting of chemotherapy adverse effects? A comparison with patient-reported symptoms from the Quality-of-Life Questionnaire C30. Journal of clinical oncology : official journal of the American Society of Clinical Oncology. 2004;22(17):3485-90.

[25] Di Maio M, Gallo C, Leighl NB, Piccirillo MC, Daniele G, Nuzzo F, et al. Symptomatic toxicities experienced during anticancer treatment: agreement between patient and physician reporting in three randomized trials. Journal of clinical oncology : official journal of the American Society of Clinical Oncology. 2015;33(8):910-5. 